\title{
Practice Spotlight: Supportive Care Research in Pediatric Oncology
}

\author{
Lee Dupuis, BSc, BScPhm, MScPhm, ACPR, FCSHP \\ Clinical Manager, Department of Pharmacy \\ Division of Haematology/Oncology \\ The Hospital for Sick Children \\ Associate Professor, Leslie Dan Faculty \\ of Pharmacy \\ University of Toronto \\ Toronto, Ontario
}

$\mathrm{E}$ ach year at The Hospital for Sick Children (also known as SickKids) in Toronto, Ontario, 300 to 350 children receive a diagnosis of cancer, and about 100 undergo bone marrow transplantation. In Canada today, more than $80 \%$ of children with cancer are cured. ${ }^{1}$ Clinicians caring for children with cancer are therefore privileged to assist children and their families as they make their cancer journey, with cure as the ultimate destination. At SickKids, children with cancer and their families join an interdisciplinary team of health care professionals in the planning and administration of their care. Pharmacists have been integral members of this team since 1991. At the time of writing, in late 2009, 9 pharmacists were involved in the clinical care of oncology and bone marrow transplant inpatients and outpatients at SickKids. A large proportion of pharmacists' interventions are intended to minimize the immediate and long-term physical and psychological effects of cancer treatment, so as to facilitate a long and normal life for the child once treatment ends.

The paradigm of pediatric oncology care is research. That is, most children with cancer (or their parents) consent to participate in research and are treated according to a research protocol. Pediatric oncology clinicians are consequently very research-focused and are generally eager to consider new research questions.

As a member of the interdisciplinary team caring for such children and as the manager responsible for the pharmaceutical care provided by the other pharmacists on the Haematology/ Oncology team, Lee Dupuis is ideally placed to ask questions about the pharmaceutical care of children with cancer. Research questions posed by Ms Dupuis are grounded in the practical aspects of care and have addressed the evaluation of new methods of using drugs such as aminoglycosides and cyclosporine, as

well as improvements in the assessment and control of treatment-related symptoms, often using drugs or interventions that are new to pediatrics. The number and diversity of patients who receive care at SickKids and the substantial amount of research-related activity that occurs routinely at the hospital create an ideal setting in which to answer these questions.

Since January 2005, Ms Dupuis's position at Sick Kids has incorporated $50 \%$ protected research time. Currently, the 2 main themes of her research program are optimizing control of chemotherapy-induced nausea and vomiting and exploring the efficacy of various pharmacokinetic targets for cyclosporine dosing for prophylaxis of graft-versus-host disease. Ms Dupuis splits the rest of her time between providing direct patient care, leading the team of Haematology/Oncology pharmacists, and coordinating the development of clinical practice guidelines.

Ms Dupuis also participates in research enterprises outside of SickKids, which have facilitated collaboration with clinicians across North America. She is chair of the Antiemetic Committee and a member of the Cancer Control Steering Committee of the Children's Oncology Group, a 300-member collaborative research organization focusing on pediatric oncology. She is also chair of the Supportive Care Committee of the Pediatric Oncology Group of Ontario (POGO) and cochair of the Standards and Guidelines Committee of $\mathrm{C}^{17}$, a network devoted to improving the care of Canadian children with cancer and hematological disorders. In these positions, not only does Ms Dupuis benefit from collaborations with researchers having similar interests, but she is also able to contribute to research programs in other institutions and to more widely influence the care of children with cancer.

Over the years, Ms Dupuis has been a member of the CSHP Education Committee and an Associate Editor of the CJHP. These experiences have served her well in her research career. They have afforded her the opportunity to network and collaborate with other researchers and to brainstorm ideas with pharmacists from various backgrounds. The CSHP Research and Education Foundation has generously supported 2 of her studies: "Preliminary Evaluation of Antiemetic Strategies for Children Receiving Antineoplastics" 2,3 and "Development of an Oral Liquid Aprepitant Formulation". ${ }^{4}$

Pharmacists in any practice area who are interested in pursuing clinical research are encouraged to leverage the oppor- 
tunities for collaboration with like-minded individuals in their own institutions and to facilitate collaboration with pharmacists and other professionals by becoming active in outside agencies such as the CSHP, the American College of Clinical Pharmacy, and other subspecialty organizations. Universities also offer brief institutes to develop skills in areas such as epidemiology, grant writing, and statistics, which are of great help to a research career. Writing skills can be honed only by preparing and submitting manuscripts for publication and by critically reviewing the comments of editors and reviewers. Pharmacists are now acknowledged as clinicians and are well placed to know what drug-related research questions are most pertinent to the care of their patients. We pharmacists are also able, by marshalling the various resources available to all clinicians, to craft well-designed studies to answer these questions.

\section{References}

1. Canadian Cancer Society, Steering Committee. Canadian cancer statistics 2009. Toronto (ON): Canadian Cancer Society; 2009.

2. Dupuis LL, Lau R, Greenberg ML. Effectiveness of strategies for preventing acute antineoplastic-induced nausea and vomiting in children with acute lymphoblastic leukemia. Can J Hosp Pharm 1999;52:350,352-361.

3. Dupuis LL, Lau R, Greenberg ML. Delayed nausea and vomiting in children receiving antineoplastics. Med Pediatr Oncol 2001;37(2):115-121.

4. Dupuis LL, Lingertat-Walsh K, Walker SE. Stability of an extemporaneous oral liquid aprepitant formulation. Support Care Cancer 2009;17(6):701-706.

Acknowledgements

I would like to take this opportunity to acknowledge the support I have received from staff at The Hospital for Sick Children: Beverley Hales, Director of Pharmacy; Judy van Clieaf, Director, Haematology/Oncology/ BMT Program; Dr. Victor Blanchette, Chief, Division of Haematology/ Oncology; and Dr. Lillian Sung, Scientist, Research Institute. I would also like to acknowledge the superb pharmaceutical care that the clinical and dispensing pharmacist and technician teams provide to patients in the Haematology/Oncology/BMT Program. Finally, I thank the children and their parents who have participated in the studies that my research team undertaken.-Lee Dupuis

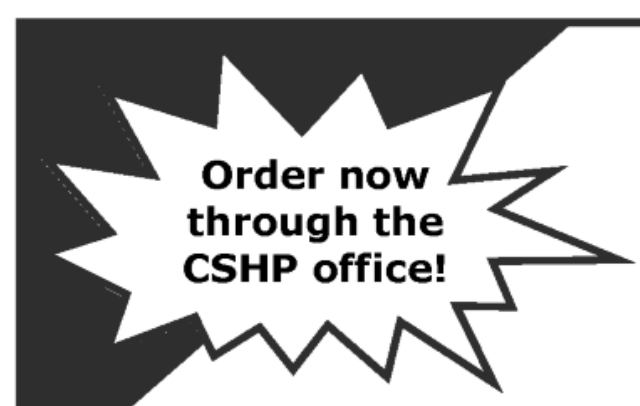

\section{Safe and Effective}

The Eight Essential Elements of an Optimal Medication-Use System

Editor: Neil J. MacKinnon, PhD, FCSHP

Medication is the most relied-upon treatment in health care today. Despite its importance, the current medication-use system suffers from problems related to lack of safety and quality. Safe and Effective explores the medication-use system and, more importantly, provides concrete and straightforward strategies for improving it.
"The patient safety movement is profoundly changing the face of modern health care. This effort by Neil MacKinnon and his capable and progressive group of colleagues is a "must read" for clinicians, practitioners, academics and students who are part of this important journey. This addition to the literature will assist in advancing patient safety practices for Canadian caregivers and will most certainly benefit those we all serve." - Phil Hassen, CEO, Canadian Patient Safety Institute

Now available to order through the CSHP office - $\$ 81$ for CSHP members and $\$ 90$ for nonmembers (plus applicable taxes and shipping). Please find our order form online at www.cshp.ca under the "Products and Publications" drop-down menu. 